\title{
Study on the Cutter Suction Dredgers Productivity Model and Its Optimal Control
}

\author{
Minghong Yao, Yanling Wang, Jing Shang and Jianyong Zhang* \\ Hohai University, Changzhou Campus, Changzhou, China \\ ${ }^{*}$ Corresponding author
}

\begin{abstract}
This Controlling the yield of cutter suction dredger is a hot spot for researchers at home and abroad, however, there is few strict mathematical models proving the existing research results, and most of the results are qualitative. In order to optimize the value of dredgers main control variables, this paper puts forward a non-linear productivity optimization model based on Lagrangian Algorithm. Taking Tianshi-Caofeidian project as an example, this paper provides the optimal value ranges of dredgers main control variables and also proves some existing experimental conclusions. After comparing experimental control values with this papers results, the experimental control values are supposed to be increased, and this result can be used for practical engineering.
\end{abstract}

Keywords-cutter suction dredger; non-linear productivity optimization model; Lagrangian

\section{INTRODUCTION}

Improving Cutter suction dredgers production has a significant influence on actual engineering. Optimization research of Cutter suction dredgers productive conditions is a hot spot at home and abroad. However, there is few strict mathematical models proving the existing research results, and most of the results are qualitative [1]. This paper puts forward a non-linear productivity optimization model based on Lagrangian algorithm to provide the optimal value ranges of dredgers main control variables and also proves some existing experimental conclusions.

\section{InTRODUCTION OF CUTTER SUCTION DREDGERS MUd PUMP SYSTEM AND PING SYSTEM}

\section{A. Work rules of Cutter Suction Dredger}

Cutter suction dredger usually use spud carriage to work [2]. Dredger is fixed with pillar in the middle of channel, and dredger can go around the pillar to remove mud by cables pull. Due to the limited channel width, the dredger will change its direction periodically. Figure 1 is dredgers GPS trajectory during working hours.
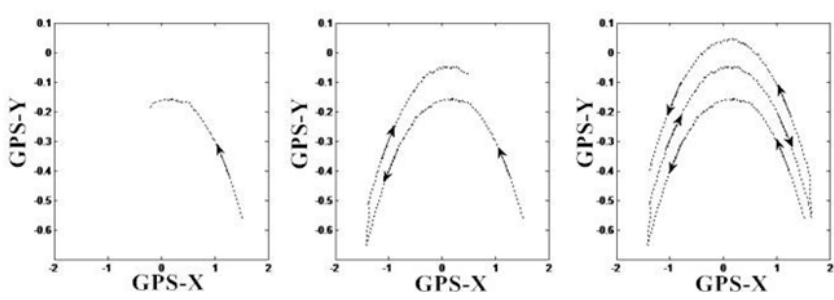

FIGURE I. DREDGERS GPS TRAJECTORY DURING WORKING HOURS.

\section{B. Pump System and Piping System}

Cutter suction dredger is mainly consist of hull, spud system, slurry transport system which includes mud pump system and pipeline system, cutter cutting system, transverse system, Electric hydraulic auxiliary system and etc.

The characteristics of centrifugal mud pump can be deduced by Euler equation [2], [3]. Following is relation between pumps theoretical pressure head and theoretical flow rate Q:

$$
H_{\text {pump }}=-k_{2} Q^{2}-\frac{k_{1} \cot \beta}{g \pi D_{2} b_{2}} u_{2} Q_{2}+\frac{k_{1} u_{2}^{2}}{g}
$$

Where $\mathrm{H}_{\text {pump }}$ denotes theoretical pressure head, $\mathrm{Q}$ denotes theoretical flow rate, $\mathrm{u}_{2}$ represents tangential velocity of pump, represents angle of vane, $b_{2}$ is width of impeller, $D_{2}$ is diameter of entrance, $K_{1}$ and $K_{2}$ is coefficient of correction, $g$ is acceleration of gravity.

There are many empirical formulas of piping systems resistance loss [4], [5]. This paper selects sliding bed and Heterogeneous suspension flow model as follows:

$$
\begin{aligned}
H_{\text {pipe }}= & \frac{8 L Q^{2}}{\pi^{2} \gamma_{s} g D^{2}\left(0.314+\mathrm{Re}^{-0.25}\right)}-\frac{33 L v_{s}\left(1-\gamma_{w} / \gamma_{s}\right) C_{f}}{0.314+\mathrm{Re}^{-0.25}} \\
& +\sum_{j=1}^{m} \frac{8 \xi_{j} Q^{2}}{g \pi^{2} D^{4}}+H_{g}
\end{aligned}
$$

Where $\mathrm{H}_{\text {pipe }}$ denotes piping systems resistance loss, $\mathrm{Q}$ denotes theoretical flow rate, $\mathrm{C}_{\mathrm{f}}$ denotes slurry concentration, $\mathrm{V}_{\mathrm{s}}$ represents terminal settling velocity, $\mathrm{s}$ represents relative 
density of slurry, w represents relative density of water, $g$ is acceleration of gravity, $\mathrm{j}$ is local loss coefficient, $\mathrm{D}$ is diameter of mud pipe, $\mathrm{H}_{\mathrm{g}}$ is altitude, $\mathrm{L}$ is length of pipeline, $\mathrm{Re}$ is Reynolds number. The parameters mentioned above are closely related to the specific machine and construction environment. There are no numerical values for reference.

\section{Introduction of Stable Operating Condition}

Random disturbing factors usually affect cutter suction dredgers operating condition. Actually, cutter suction dredgers operating condition tends to stabilize gradually. Engineers suppose that the operating condition get stable. This paper will concentrate on those stable operating conditions for further study. Cutter suction dredger stable operating condition is determined by cross point of the mud pump characteristic curve and the intersection of the pipeline characteristic curve [6], and this conclusion is basis for selecting stable operating conditions, i.e. $\mathrm{H}_{\text {pump }}=\mathrm{H}_{\text {pipe }}$, the physical meaning of this equation is that the pressure head provided by the pump for slurry is balanced with the head consumed by the pipeline.

\section{DETERMINATION OF THE UNKNOWN PARAMETERS OF Slurry TRANSPort System Based ON THE Clems LAW}

As mentioned above, the parameters of the mud transport system are closely related to the specific construction project. In this section, the unknown parameters of the dredge slurry transport system under the construction environment of Tianshi-Caofeidian Project will be solved correctly According to the control equation $\mathrm{H}_{\text {pump }}=\mathrm{H}_{\text {pipe }}$, we can get following equation:

$$
\begin{aligned}
& \frac{8 L Q^{2}}{\pi^{2} \gamma_{s} g D^{2}\left(0.314+\mathrm{Re}^{-0.25}\right)}-\frac{33 L v_{s}\left(1-\gamma_{w} / \gamma_{s}\right) C_{f}}{0.314+\mathrm{Re}^{-0.25}}+\sum_{j=1}^{m} \frac{8 \xi_{j} Q^{2}}{g \pi^{2} D^{4}} \\
& +H_{g}=-K_{2} Q^{2}-\frac{K_{1} \cot \beta}{g \pi D_{2} b_{2}} u_{2} Q_{2}+\frac{K_{1} u_{2}^{2}}{g}
\end{aligned}
$$

After combining some unknown parameters, we can get following equation:

$$
A Q^{2}+B u_{2} Q+C C_{f}-D u_{2}^{2}+E=0
$$

Where:

$$
\begin{gathered}
A=\frac{8 L}{\pi^{2} \gamma_{s} g D^{5}\left(0.314+\mathrm{Re}^{-0.25}\right)}+\sum_{j=1}^{m} \frac{8 \xi_{j}}{g \pi^{2} D^{4}}+K_{2} \\
B=\frac{K_{1} \cot (\beta)}{g \pi D_{2} b_{2}} \quad C=\frac{33 L v_{s}\left(1-\gamma_{w} / \gamma_{s}\right) C_{f}}{0.3164+\mathrm{Re}^{-0.25}} \quad D=\frac{K_{1}}{g} \quad E=H_{g}
\end{gathered}
$$

In all stable operating conditions, a large number of data of flow conditions, mud concentration and mud pump tangential flow velocity can be used to make up the operating conditions vector $\vec{b}=\left[\begin{array}{lllll}Q^{2} & u_{2} \cdot Q & C_{f} & -u_{2}^{2} & 1\end{array}\right]$. By defining a parameter vector as $\vec{a}=\left[\begin{array}{lllll}A & B & C & D & E\end{array}\right] . \vec{a}$ and $\vec{b}$ are vectors in the R5 space, i.e:

$$
A^{*} Q^{2}+B^{*} u_{2} Q+C^{*} C_{f}-D^{*} u_{2}{ }^{2}+E^{*} 1=0
$$

The equation implies that the vector $\vec{a}$ and $\vec{b}$ are "perpendicular" to each other in R5 space. In this paper, we select multiple sets of operating condition vector group $\left\{\vec{b}_{i} \mid i=1,2,3,4\right\}$ vector a by the following equation:

$$
\left\{\begin{array}{l}
\left(Q^{2}\right)_{1} * \frac{A}{D}+\left(u_{2} Q\right)_{1} * \frac{B}{D}+\left(C_{f}\right)_{1} * \frac{C}{D}+\frac{E}{D}=\left(u_{2}^{2}\right)_{1} \\
\left(Q^{2}\right)_{2} * \frac{A}{D}+\left(u_{2} Q\right)_{2} * \frac{B}{D}+\left(C_{f}\right)_{2} * \frac{C}{D}+\frac{E}{D}=\left(u_{2}^{2}\right)_{2} \\
\left(Q^{2}\right)_{3} * \frac{A}{D}+\left(u_{2} Q\right)_{3} * \frac{B}{D}+\left(C_{f}\right)_{3} * \frac{C}{D}+\frac{E}{D}=\left(u_{2}^{2}\right)_{3} \\
\left(Q^{2}\right)_{4} * \frac{A}{D}+\left(u_{2} Q\right)_{4} * \frac{B}{D}+\left(C_{f}\right)_{4} * \frac{C}{D}+\frac{E}{D}=\left(u_{2}^{2}\right)_{4}
\end{array}\right.
$$

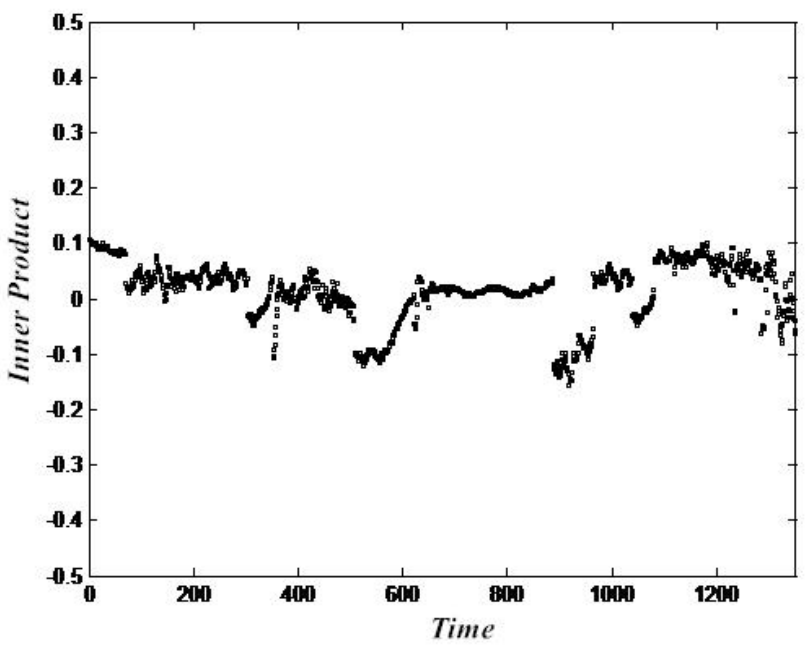

FIGURE II. THE INNER PRODUCT OF DATA USING FOR VERIFYING

$$
\left[\begin{array}{c}
(A / D)_{j} \\
(B / D)_{j} \\
(C / D)_{j} \\
(E / D)_{j}
\end{array}\right]=\left[\begin{array}{llll}
\left(Q^{2}\right)_{1} & \left(u_{2} \cdot Q\right)_{1} & \left(C_{f}\right)_{1} & 1 \\
\left(Q^{2}\right)_{2} & \left(u_{2} \cdot Q\right)_{2} & \left(C_{f}\right)_{2} & 1 \\
\left(Q^{2}\right)_{3} & \left(u_{2} \cdot Q\right)_{3} & \left(C_{f}\right)_{3} & 1 \\
\left(Q^{2}\right)_{4} & \left(u_{2} \cdot Q\right)_{4} & \left(C_{f}\right)_{4} & 1
\end{array}\right]^{-1} *\left[\begin{array}{l}
\left(u_{2}^{2}\right)_{1} \\
\left(u_{2}^{2}\right)_{2} \\
\left(u_{2}^{2}\right)_{3} \\
\left(u_{2}{ }^{2}\right)_{4}
\end{array}\right]
$$

After proper screening, the determinant of the coefficient matrix of the system has a unique solution according to the Cramer's law. Consider the actual measurement error, many parameters are not all parallel to the supper plane spanned by 
these vectors, forming a small deviation to each other. The solution here is to take the average of many $\overrightarrow{a_{j}}$. This paper uses Matlab to select 500 sets of data, calculate the average of $\overrightarrow{a_{j}}$, which is noted by $\vec{a}=\sum_{j=1}^{500} \frac{\vec{a}_{j}}{500}$. The calculation results are as follows:

$$
\left[\overline{\frac{(A / D)}{(B / D)}}\left[\frac{\overline{(C / D)}}{\overline{(E / D)}}\right]=\left[\begin{array}{c}
\sum_{j=1}^{500}\left(\frac{A}{500 D}\right)^{j} \\
\sum_{j=1}^{500}\left(\frac{B}{500 D}\right)^{j} \\
\sum_{j=1}^{500}\left(\frac{C}{500 D}\right)^{j} \\
\sum_{j=1}^{500}\left(\frac{E}{500 D}\right)^{j}
\end{array}\right]=\left[\begin{array}{c}
0.4912 \\
-0.8683 \\
0.0016 \\
0.0694
\end{array}\right]\right.
$$

In order to verify the reasonableness of the parameter vector, we choose 1400 sets of data to construct 1400 independent operating condition vectors, again using Matlab. And the calculated parameters vector is respectively calculated with 1400 independent operating condition vectors. As is shown in figure 2, the result of the product fluctuates up and down at value zero, which means that the solution of parameter vector has a strong rationality.

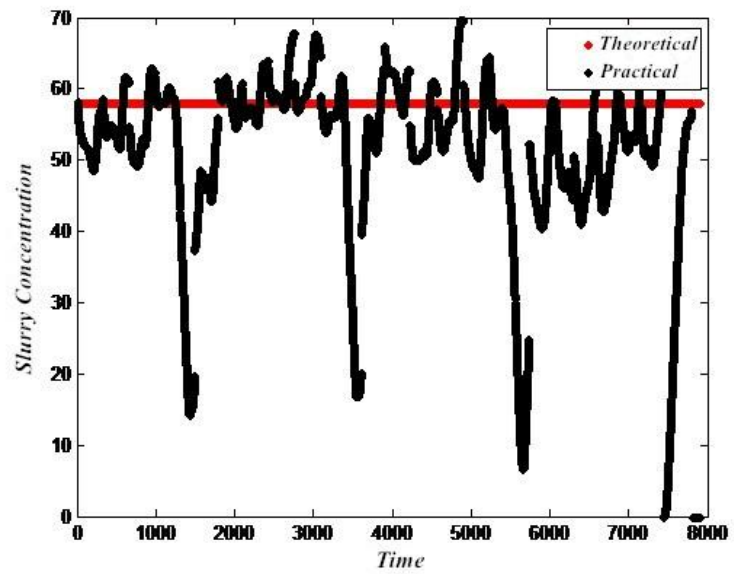

FIGURE III. THE VALIDATION OF RESULTS

\section{Productivity Optimization Model Based ON LAGRANGIAN ALGORITHM}

We are committed to discuss optimization of cutter-suction dredger's control variables for a high-yield target, i.e. what we want is, the most value of the objective function $\mathrm{P}=\mathrm{Q} * \mathrm{Cf}$ [7], [8], where $\mathrm{P}$ is the output per unit time, following is mathematical statement of this problem:

$$
\begin{aligned}
& \max \quad P=Q C_{f} \\
& \text { s.t }\left\{\begin{array}{l}
A Q^{2}+B u_{2} Q+C C_{f}+D u_{2}{ }^{2}+E=0 \\
0 \leq u_{2} \leq u_{2 \max } \\
0 \leq C_{f} \leq 1
\end{array}\right.
\end{aligned}
$$

Construct the Lagrangian auxiliary function based on the Lagrange multiplier method as follows:

$$
L=Q C_{f}+\lambda\left(\overline{(A / D)} Q^{2}+\overline{(B / D)} u_{2} Q_{+} \overline{(C / D)} C_{f}-u_{2}^{2}+\overline{(E / D)}\right)
$$

Where:

$$
\begin{array}{ll}
\overline{(A / D)}=0.4192 & \overline{(B / D)}=-0.8683 \\
\overline{(C / D)}=0.0016 & \overline{(E / D)}=0.0694
\end{array}
$$

Resolving the stationary point of the function $L\left(Q, C_{f}, u_{2}, \lambda\right)$ by the following equations:

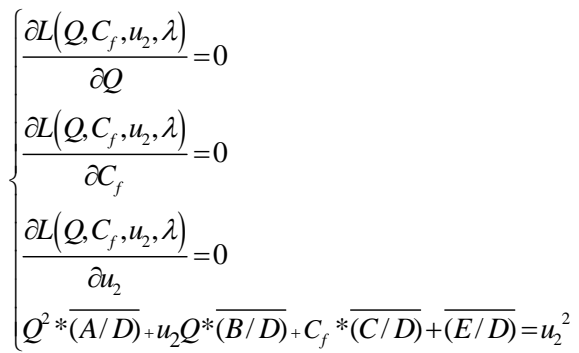

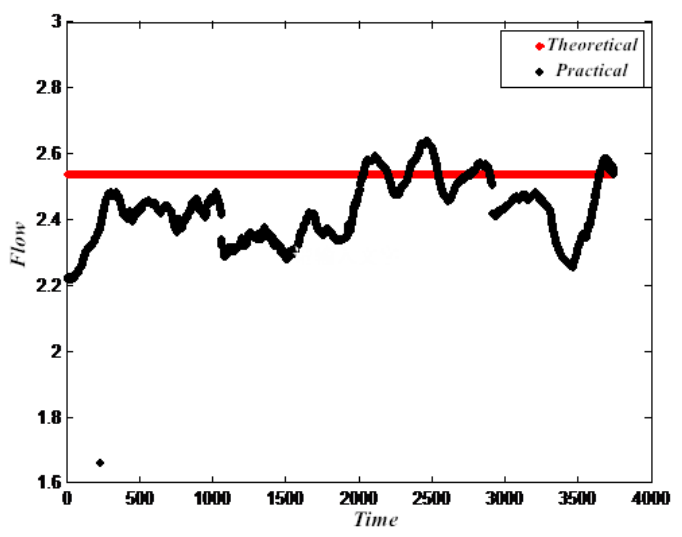

FIGURE IV. THE VALIDATION OF RESULTS

The equations have no real solution, i.e. the objective function has no extreme value point. It shows that the maximum point is the boundary point of the constraint, so we will confirm where to get the point by the following analysis. 
This paper makes the partial derivatives on both sides of the constraint condition Hpump=Hpipe, and by analyzing the numerical properties of related parameters [9], we get following results:

$$
\begin{gathered}
\frac{\partial Q}{\partial u_{2}}=\frac{\frac{K_{1}}{g}\left(2 u_{2}-\frac{\cot (\beta)}{g \pi D_{2} b_{2}} Q\right)}{\frac{\partial H_{\text {pipe }}}{\partial Q}+2 K_{2} Q+\frac{K_{1} \cot (\beta)}{g \pi D_{2} b_{2}} u_{2}} \geq 0 \\
\frac{\partial Q}{\partial C_{f}}=\frac{-\frac{\partial H_{\text {pipe }}}{\partial C_{f}}}{\frac{\partial H_{\text {pipe }}}{\partial Q}+2 K_{2} Q+\frac{K_{1} \cot (\beta)}{g \pi D_{2} b_{2}} u_{2}} \leq 0
\end{gathered}
$$

Assuming the pipe slurry concentration is certain, the yield is max, when $u 2=u 2 \max$, i.e. dredge pump's speed $\mathrm{n}$ is max within an allowable range for equipment. And if the dredge pump's speed is certain, we can conclude the greater the slurry concentration, the smaller the pipe flow.

As we can see, the conclusion is consistent with that got by the empirical optimization method in Wang Qisong's paper [8] However, we verify the conclusion with specific mathematical model from mathematical perspective. And we can find that $P_{\max }=Q\left(u_{2 \max }, C_{f}{ }^{*}\right) \cdot C_{f}{ }^{*}$, i.e. the relation of $\operatorname{Pmax}(\mathrm{Cf})$ and slurry concentration $\mathrm{Cf}$ is not the direct or inverse proportion. The cutter suction dredger system has the optimal solution to maximize the yield per unit time. The problem has been transformed into solving the single variable extreme of slurry concentration. Equations are as follows:

$$
\left\{\begin{array}{l}
\frac{\partial P}{\partial C_{f}}=Q\left(u_{2}, C_{f}\right)+\frac{\partial Q\left(u_{2}, C_{f}\right)}{\partial C_{f}} C_{f}=0 \\
H_{\text {pump }}=H_{\text {pipe }} \\
u_{2}=u_{2 \max }
\end{array}\right.
$$

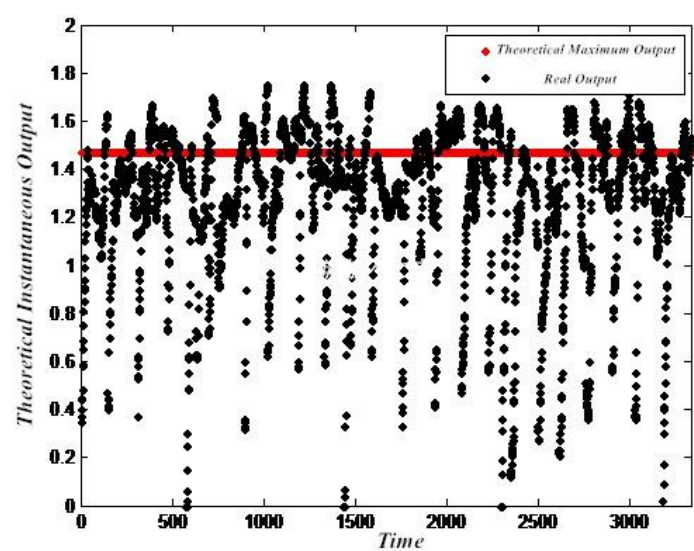

FIGURE V. THE VALIDATION OF THEORETICAL INSTANTANEOUS OUTPUT
By solving the equations, we can get following results:

$$
C_{f}^{*}=58 \% \quad Q^{*}=2.54 m^{3} / s \quad u_{2}^{*}=57.5 \mathrm{rad} / \mathrm{s}
$$

With further solution we also get:

$$
P_{\max }=Q\left(u_{2 \max }, C_{f}^{*}\right) * C_{f}^{*}=2.54 * 0.58=1.47 \mathrm{~m}^{3} / \mathrm{s}
$$

\section{ApPLICATION Of THEORETICAL ANALYSIS}

We select the data of stable operating conditions in specific project to compare the theoretical value with actual value of slurry density and flow rate in figure 3 and figure 4

We select the data of stable operating conditions in specific project and figure out the theoretical maximum yield per unit time, and then we compare its theoretical value with actual value in figure 5 . As we can see, the theoretical maximum is greater than the most actual data, so the result is reasonable.

In practical engineering, most of us change the mud pump speed $\mathrm{n}$, reamer transverse moving speed $\mathrm{Vt}$, reamer rotating speed $\mathrm{Vr}$ and dredging depth $\mathrm{h}$ to control the work [1]. The flow and the slurry density in tubes are observed quantities for reference. In the previous sections, in order to get the maximum yield per unit time, the upper limit value of dredge pump nmax should be set. And we figure out $\mathrm{Pmax}=1.47 \mathrm{~m} 3 / \mathrm{s}$, select all the stable points which meet $P \in[1.44,1.50]$ to form the set $\Omega_{1}$. Since the yield per unit time of points in $\Omega_{1}$ is max, it's feasible to determine the values of control variables by analyzing the points in $\Omega_{1}$. Through the statistics of control variables' numerical properties in $\Omega_{1}$, we conclude the control interval of main control variables as Table I:

TABLE I: OPTIMAL VALUE RANGES OF VARIABLES

\begin{tabular}{cc}
\hline Control variable & Value range \\
\hline reamer transverse moving speed & {$[0.16,0.21]$} \\
$(\mathrm{m} / \mathrm{s})$ & \\
reamer rotating speed $(\mathrm{rad} / \mathrm{s})$ & {$[2.76,2.90]$} \\
dredging depth $(\mathrm{m})$ & {$[15.43,15.79]$} \\
\hline
\end{tabular}

\section{CONCLUSION}

Improving cutter suction dredger's production has a significant influence on actual engineering. Cutter suction dredger's construction control is closely related to the specific machine and construction environment. At present, there is few strict mathematical models proving the existing research results at home and abroad, and most of them are qualitative. This paper puts forward the non-linear productivity optimization model based on Lagrangian algorithm to provide the optimal value ranges of dredger's main control variables and also proves some existing experimental conclusions. However, there is still a huge research space for improving the control value's precision. 


\section{REFERENCES}

[1] Tang Jianzhong, Wang Qingfeng, Bi Zhiyue.Model and optimization method for dredging operations[J]. Journal of Zhejiang University (Engineering Science).2008,(5):850-857.

[2] Bree,S.E.M.Centrifugal Dredge pumps.IHC Holland.2003.

[3] Miedema,S.A.Modeling and Simulation of the Dynamic Behavior of a Pump/Pipeline System.17th Annual Meeting and Technical Conference of the Western Dredging Association,New Orleans,June 1996.

[4] Matousek,V. Flow mechanism of sand water mixture in pipeline. Doctoral Thesis, Delft University Press, 1997.

[5] Matousek, V.Flow friction of mixture composed of fine sand and coarse sand.Proc.The 4th Int.Conf.On Multiphase Flow.New Orleans, 2001.

[6] Li Yan, Li Zhiqiang. Dredging optimization of Cutter suction dredger.[J]. Science Technology and Engineering.2011, (6):1218-1221.

[7] Tang Jianzhong. Study on cutter suction dredging operation optimization and its control research[D]. Machinery and energy engineering college of Zhejiang University, 2007.

[8] Wang Qisong, Yan Jun, Deng Jiaquan. Study on high yield of cutter suction dredger[J]. Port and Waterway Engineering.2013, (3):48-55.

[9] Wang Hairong, He Yanping. Analysis of Methods for Calculating Flow Resistance in Dredging Pipelines[J]. China Harbour Engineering.2008,(5):17-20. 